\title{
The stellar magnetic dynamo during the evolution across the main sequence
}

\author{
Swetlana Hubrig \\ Astrophysikalisches Institut Potsdam \\ An der Sternwarte 16 \\ 14482 Potsdam, Germany \\ email: shubrig@aip.de
}

\begin{abstract}
In comparison with stellar magnetic field studies in the eighties and nineties, where most effort was focused on A, B, and active solar-type stars, magnetic fields are currently directly measured in massive early $\mathrm{B}$ and $\mathrm{O}$ stars with radiative envelopes, as well as in the lower mass tail consisting of fully convective late-M dwarfs. Knowledge of the magnetic field topology of stars of different mass and at different evolutionary stages is important to understand the underlying magnetic field generation mechanisms. I review the present status of magnetic field studies along with the results of theoretical modeling.
\end{abstract}

Keywords. MHD, instabilities, techniques: polarimetric, binaries: spectroscopic, stars: chemically peculiar, stars: evolution, stars: formation, stars: magnetic fields, stars: pre-main-sequence, stars: spots

\section{Introduction}

The presence of a convective envelope is a necessary condition for significant magnetic activity. Magnetic activity is found all the way from the late A-type stars (e.g. in Altair: Robrade \& Schmitt 2009) with very shallow convective envelopes down to the coolest fully convective M-type stars. Among the early-type main sequence A and B stars, fields with large-scale organised structure consistent with the oblique rotator model have been detected in the subgroup of the Ap and Bp stars. Although these stars have the best studied fields among the early-type stars, the origin of their magnetic fields is still poorly understood. The presence of magnetic fields in massive early B and $\mathrm{O}$ stars is known in a very small sample only, but advances in instrumentation over the past decade have led to magnetic field detections in a gradually growing subset of massive stars, which frequently present cyclic wind variability, $\mathrm{H} \alpha$ emission variations, non-thermal radio/X-ray emission and transient features in the absorption line profiles.

\section{The presence of a convection zone in massive stars}

Recent theoretical developments have highlighted the potentially significant influence of magnetic fields on the structure, evolution and environment of massive stars. The presence of a convection zone in the outer envelope of hot massive stars was recently studied by Cantiello et al. (2009). The authors used a stellar evolution code to compute a grid of massive star models at different metallicities. A small convection zone in the envelopes of sufficiently luminous massive main-sequence stars due to the so-called iron peak in stellar opacities was previously predicted by Slothers \& Chin (1993).

Cantiello et al. mapped the strength of the iron convection zone (FeCZ) in the H-R diagram showing its prominence as a function of stellar parameters. Since in their models the FeCZ has a spatial extent similar to the solar convection zone, the authors suggest 


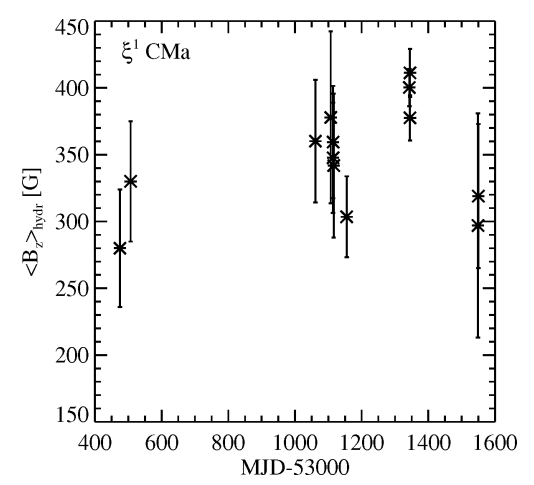

Figure 1. Magnetic field measurements for one of the hottest $\beta$ Cep star, $\xi^{1} \mathrm{CMa}$, during the last 4.4 years.
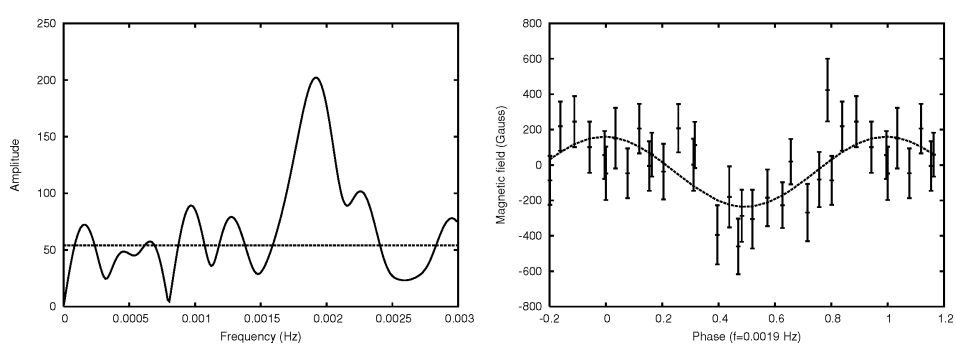

Figure 2. A study of the magnetic field of the B0.2V star $\theta$ Car. Left: Fourier spectrum for the magnetic field data. The horizontal line gives the noise level. Right: Phase diagram for the same dataset for the best candidate frequency of $0.0019 \mathrm{~Hz}$, i.e. about 8.8 minutes.

that a dynamo may also work in rapidly rotating OB stars. The rise time of buoyant magnetic flux tubes from the FeCZ to the stellar surface will then be then much shorter than in the model by MacGregor \& Cassinelli (2003), who considered the rise of flux tubes from the convective core. The interaction of the stellar wind with the localized surface magnetic field could increase the loss of stellar angular momentum and could probably explain co-rotating density patterns in the outflowing wind. No definitive magnetic field was ever detected in Wolf-Rayet stars and currently only half a dozen of $\mathrm{O}$ stars have published magnetic fields (e.g., Donati et al. 2002; Donati et al. 2006a; Hubrig et al. 2008a). Among the hottest early B-type stars, a weak $(\sim 40 \mathrm{G})$ magnetic field has been discovered in the B0.2V star $\tau$ Sco (Donati et al. 2006b) and in one of the hottest $\beta$ Cephei stars, the B0.7IV star $\xi^{1} \mathrm{CMa}$, with a rather large longitudinal magnetic field of up to $400 \mathrm{G}$ (Hubrig et al. 2006a). The amplitude of the observed magnetic field variations in the B0.7IV star $\xi^{1} \mathrm{CMa}$ is rather low: The obtained values of the longitudinal magnetic field are in the range from 280 to $410 \mathrm{G}$ (see Fig. 1). Our search for a variation period of the magnetic field in this star using a Fourier analysis did not reveal any significant frequency (Hubrig et al. 2009a).

The results of the search for a magnetic field in another hot star, $\theta$ Car, with spectral type B0.2V, using FORS 1 at the VLT were rather inconclusive (Hubrig et al. 2008b). To monitor the behaviour of the magnetic field over at least a part of the stellar surface, we carried out a time series of exposures with short integration time over the time span of $\sim 1.2 \mathrm{~h}$. The observed magnetic field changed several times from positive to negative values over the observing time span. To find the period of variations of the magnetic 


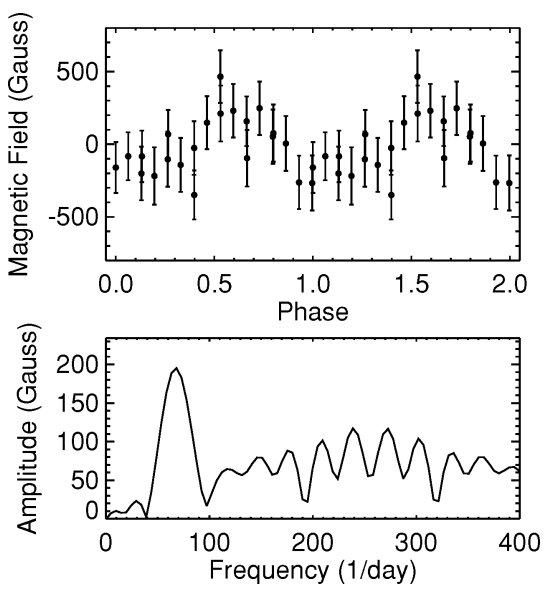

Figure 3. Phase diagram and amplitude spectrum for the magnetic field measurements of the Be star $\lambda$ Eri in August 2006.

field, we performed a Fourier analysis of the magnetic field measurements. The Fourier spectrum with an amplitude of the magnetic field variation of $202 \pm 43$ Gauss and the phase diagram for $f=0.0019 \mathrm{~Hz}$ are presented in Fig. 2, indicating a possible cyclic variability of the magnetic field.

A number of physical processes in classical Be stars (e.g., angular momentum transfer to a circumstellar disk, channeling stellar wind matter, accumulation of material in an equatorial disk, etc.) are more easily explainable if magnetic fields are invoked. For ten Be stars we performed time-resolved magnetic field measurements over one hour (corresponding to a time series of 20 to 30 measurements per star; Hubrig et al. 2009b). In this way, similar to the study of $\theta$ Car, we obtained information on the behaviour of the localized transient magnetic field over a part of the stellar surface. In the obtained amplitude spectra a $2.4 \sigma$ peak corresponding to a period of $21.12 \mathrm{~min}$ was detected in the data set of measurements carried out using hydrogen lines in the Be star $\lambda$ Eri (see Fig. 3). Our observations indicate that a strong field could possibly exist locally, but with a topology such that its net effect can appear only sporadically in disk-integrated variations. Our time-resolved magnetic field measurements of the remaining Be stars indicate that four other Be stars may display a magnetic cyclic variability on a time scale of minutes or tens of minutes. However, since the mechanism of the generation and maintenance of magnetic fields in massive stars is not well understood yet, we are cautious in drawing any conclusion on the behaviour of a magnetic field in $\theta$ Car or the studied Be stars.

\section{Stars of spectral type B6-A0 with $\mathrm{HgMn}$ peculiarity and Ap/Bp stars with large-scale organized magnetic fields}

Our recent studies of spectroscopic binaries with B6-A0-type suggest that the majority of slowly rotating late B-type stars formed in binary systems with certain orbital parameters become HgMn stars. The aspect of inhomogeneous distribution of some chemical elements over the surface of HgMn stars has been, for the first time, discussed by Hubrig \& Mathys (1995) and has been confirmed in recent studies (e.g. Hubrig et al. 2006b). In Fig. 4 we present a typical Doppler Imaging reconstruction of $\mathrm{Y}$ on the surface of a HgMn star, in this case the eclipsing SB2 HgMn binary AR Aur (e.g. Savanov et al. 2009). 

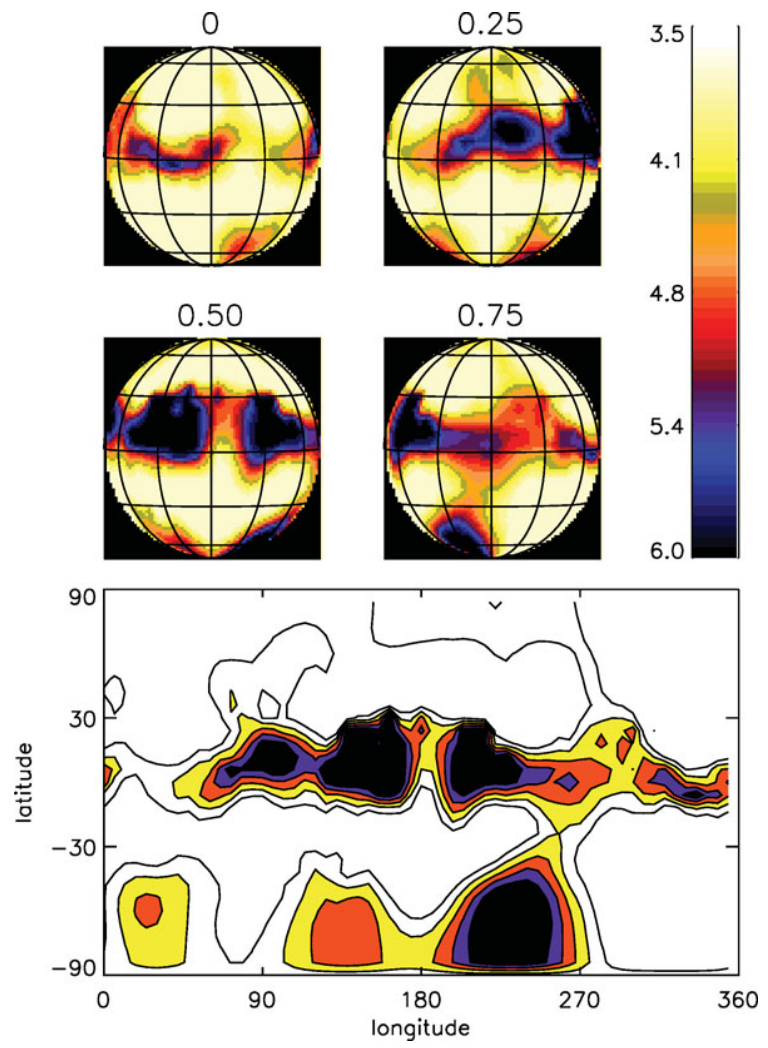

Figure 4. Results of the Doppler Imaging reconstruction of $\mathrm{Y}$ on the surface of the eclipsing binary AR Aur. Spherical Doppler Imaging maps are presented at four equidistant rotational phases (upper panel). The rectangular plot (lower panel) shows a pseudo-Mercator projection of the surface maps.

The distribution of some elements was found to present fractured rings around the stellar rotational equator with some features close to the rotational pole.

For one SB1 system, HD 11753, it was possible to gather a large number of spectra with the CORALIE echelle spectrograph attached to the $1.2 \mathrm{~m}$ Leonard Euler telescope on La Silla in Chile. In total, we obtained 113 spectra at a spectral resolution of 50,000 (Briquet et al., in preparation). Also for this system many spectral lines exhibit clear variations. In Fig. 5 we present stacked images combining the observed spectra in phase bins with steps of 0.04 .

It is a fundamental question whether magnetic fields play a significant role in the development of anomalies in binary B-type stars. Answering this question is also important for the understanding of the processes taking place during the formation and evolution of B stars in general. A scenario how a magnetic field can be built up in binary systems has been presented some time ago by Hubrig (1998) who suggested that a tidal torque varying with depth and latitude in a star induces differential rotation. Differential rotation in a radiative star can be prone to magneto-rotational instability (MRI). Magnetohydrodynamical simulations by Arlt et al. (2003) revealed a distinct structure for the magnetic field topology similar to the fractured elemental rings observed on the surface of HgMn stars. The initial model differential rotation was hydrodynamically stable (Taylor-Proudman flow), but the introduction of a magnetic field excites the MRI on a very short time-scale compared to the time-scale of microscopic magnetic diffusion. 

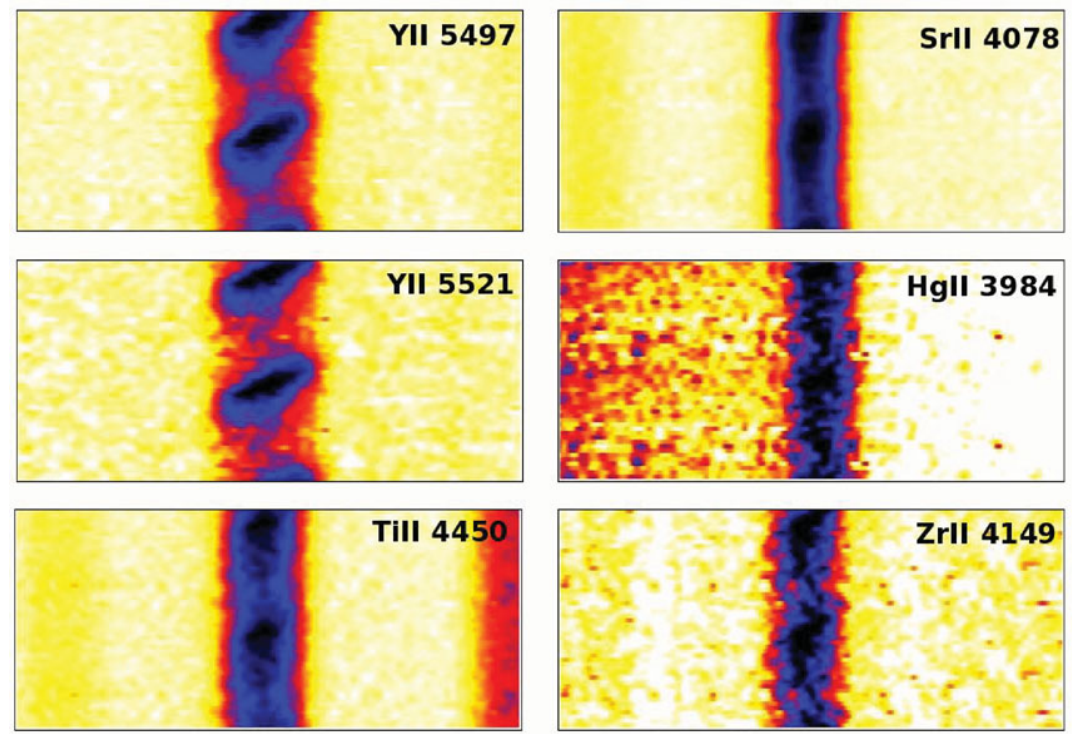

Figure 5. Variations of line profiles of different elements in the CORALIE spectra of the SB1 system HD 11753 as a function of rotation phase.

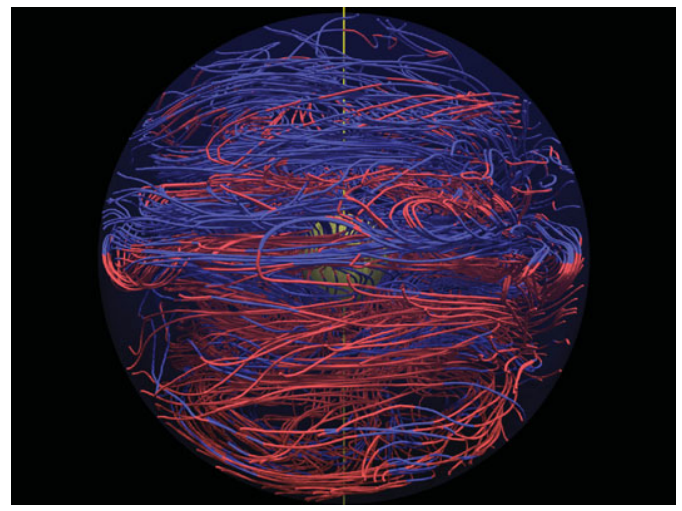

Figure 6. 3-D simulation of magnetic field lines after the onset of MRI. Blue shows radial magnetic field $\mathrm{B}_{\mathrm{r}}<0$, while red stands for $\mathrm{B}_{\mathrm{r}}>0$. Courtesy: R. Arlt.

Although the fields are not very strong, complex surface patterns can be obtained from the nonlinear, nonaxisymmetric evolution of the MRI. In Fig. 6 we present a 3-D simulation of magnetic field lines after the onset of MRI.

The magnetic fields of Ap and Bp stars have a simple non-axisymmetric topology and exhibit virtually no intrinsic variability on short and long time scales. The origin of the magnetic fields of $\mathrm{Ap}$ and $\mathrm{Bp}$ stars and details of their peculiar properties are still mysterious in spite of large efforts in their study. It is not clear yet whether their magnetism is related to the fossil magnetic fields from the interstellar medium, or results from some dynamo action capable of generating fields in radiative zones. Rüdiger \& Kitchatinov (2009) argued that non-axisymmetric poloidal fields of Ap and Bp stars can result from a kink-type instability of their internal toroidal fields. Using this mechanism, they explain the relative slow rotation and observational low boundary of magnetic field strengths of these stars. Featherstone et al. (2007) used detailed 3-D simulations to study 
the interaction of a primordial twisted magnetic field in the radiative envelope with the core-dynamo operating in the interior of an A-type star. They conclude that the interplay between such a field and the stellar dynamo can lead to the development of an organized large-scale magnetic structure.

\section{Activity in the Sun and solar-type dwarfs, evolved single stars, and fully convective stars}

It has been long assumed that solar-type stars lose their angular momentum via a magnetized wind, spin down, and become less active during their main sequence phase (e.g., Skumanich 1972; Soderblom et al. 1991). Gyrochronology relations (curves in colourperiod-age space) were introduced by Barnes (2007) and improved upon by Mamajek \& Hillebrand (2008). The rotation rate can also be tied to dynamo strength via the Rossby number, which is observationally defined as the rotation period divided by an estimate of the local convective turnover time just above the convective-radiative boundary (Noyes et al. 1984). By combining a gyrochronology relation with the $\log \mathrm{R}_{H K}^{\prime}-$ Rossby number correlation, one can predict activity as a function of colour and age (Mamajek \& Hillebrand 2008). Another useful empirical relation involves a strong correlation between coronal X-ray activity and rotation via the Rossby number (e.g. Hempelmann et al. 1995). One of the goals of research on stellar magnetic activity is to understand, from a theoretical point of view, how these empirical relations constrain stellar dynamo theory. Despite considerable efforts in theoretical stellar dynamo studies, there are still fundamental open questions concerning the deep meaning of these relations (e.g. Montesinos et al. 2001).

In contrast to solar-type stars, the $\mathrm{Ca}$ II $\mathrm{H}+\mathrm{K}$ emission from evolved single stars with low mass is weak. The drop in chromospheric emission from giants with decreasing $\mathrm{T}_{\text {eff }}$ corresponds to an even sharper drop in emissions from the transition region and corona. This has led to the discovery of so-called dividing lines in the H-R diagram. HST imaging of the M2 supergiant Betelgeuse has shown a bright spot in ultraviolet (Mg II H\&K) wavelengths (Gilliland \& Dupree 1996), i.e. located in the star's dynamic chromosphere above the photosphere. It was the first direct detection of stellar surface inhomogeneity on a star other than the Sun. Also some other observations, such as maser polarization in circumstellar envelopes of AGB stars (e.g. Sivagnanam 2004) or X-ray emission observed from some cool giant stars (e.g. Hünsch et al. 1998) suggest the existence of magnetic activity in late-type giants. The first systematic search for the presence of magnetic fields in bright giants of different spectral types and with different X-ray luminosities was carried out $\sim 15$ years ago by Hubrig et al. (1994) who reported $3 \sigma$ detections for four giants. In the last years, using the LSD technique, Konstantinova-Antova et al. (2008) detected Zeeman signatures of the magnetic field in several other giants. Numerical MHD simulations of non-linear dynamo action in Betelgeuse were presented by Dorch (2004). Three different modes of dynamo action were recognized in his computations, but it was not possible to conclude on the presence of a magnetic field since such a field is not observed.

Recent observations of active mid-M dwarfs confirmed that dynamo processes in fully-convective stars with masses of about $0.3 \mathrm{M}_{\odot}$ are very successful at generating strong poloidal fields with simple axisymmetrical configurations (e.g. Morin et al. 2008). For lower mass stars strong and simple large-scale fields were detected, while some stars display weaker and more complex magnetic field topologies. In Fig. 7 we present $\alpha \Omega$ dynamo simulations applied to the rapidly rotating fully convective M4 dwarf V374 Peg, for which an axisymmetric field was reported (M. Küker, private communication). 

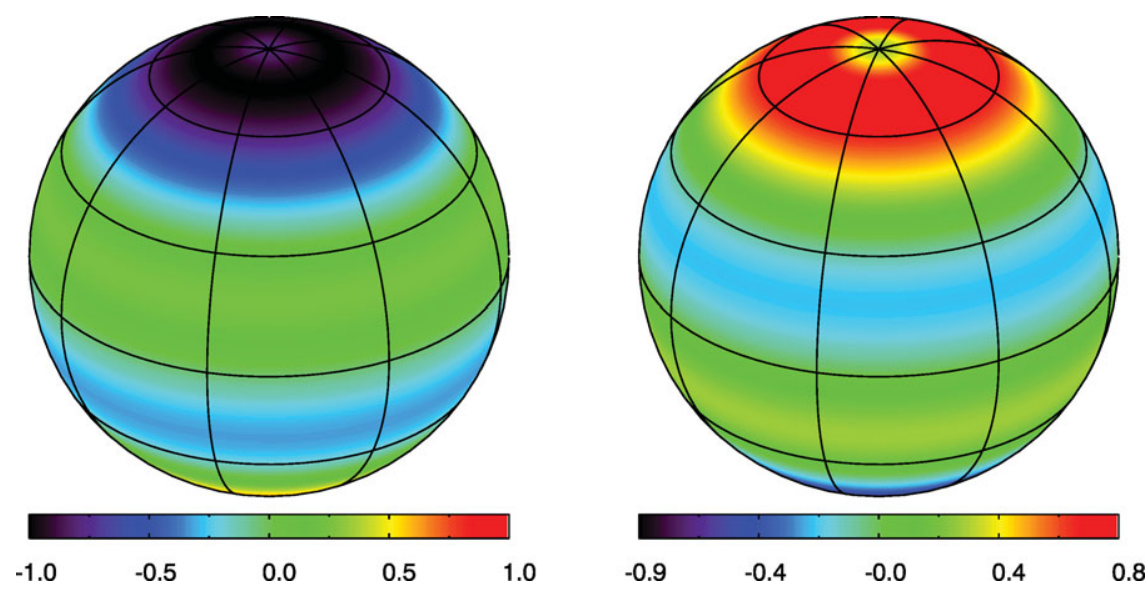

Figure 7. $\alpha \Omega$ dynamo model simulations: The star has solar-type differential rotation, i.e. it rotates faster at the equator than at the poles. The resulting magnetic field is axisymmetric and (essentially) dipolar. This model can be applied to the rapidly rotating fully convective M4 dwarf V374 Peg, for which an axisymmetric field was reported. Courtesy: M. Küker.

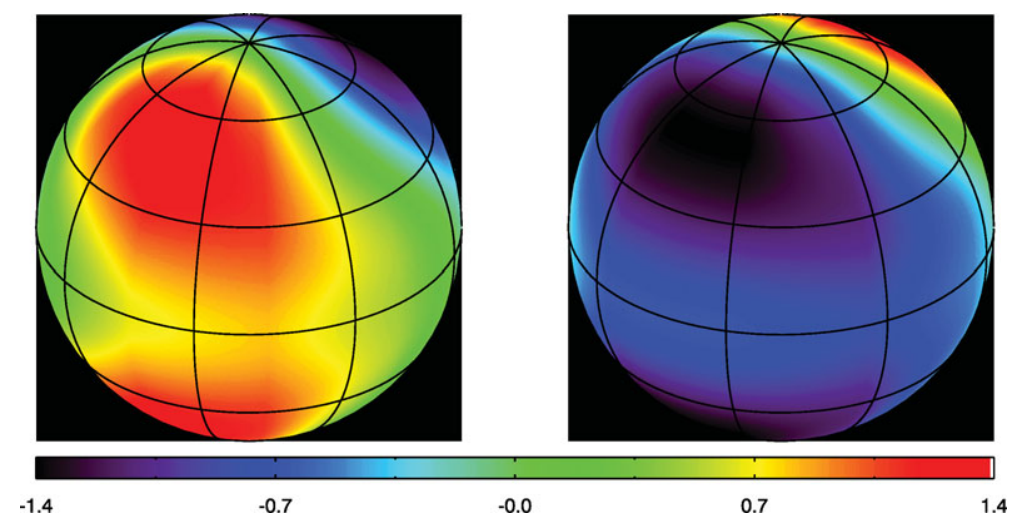

Figure 8. $\alpha^{2}$-dynamo model simulations: This dynamo works without differential rotation. All field components are generated by the helicity of the convective gas motions ( $\alpha$-effect). This type of dynamo does not prefer axisymmetric fields and the presented field is purely non-axisymmetric. The surface field shows large regions of strong vertical fields. Courtesy: M. Küker.

Large-scale magnetic topologies and surface differential rotation seem to change at spectral type $\sim \mathrm{M} 3.5$ at the boundary to full convection (e.g. Reiners \& Basri 2009). 3-D nonlinear MHD simulations of the interiors of fully convective M-dwarfs of about $0.3 \mathrm{M}_{\odot}$ were recently presented by Browning (2008). Despite of the lack of strong differential rotation, the magnetic fields realized in his simulations possess significant axisymmetric components attributed to the strong influence of rotation on the slowly overturning flows.

\section{Magnetic fields in star formation: $\mathbf{T}$ Tau stars and Herbig Ae stars}

Magnetic fields are important ingredients of the star formation process (e.g. McKee \& Ostriker 2007). Accumulated direct and indirect evidence points to a much higher level activity in the young Sun (e.g. meteoritic traces and studies of young solar analogs). $\mathrm{T}$ Tauri stars stand out by their very large emissions in the chromospheric and transitionregion lines. A model for the generation of magnetic fields in fully convective pre-main sequence stars was presented by Küker \& Rüdiger (1999), Chabrier \& Küker (2006), and 

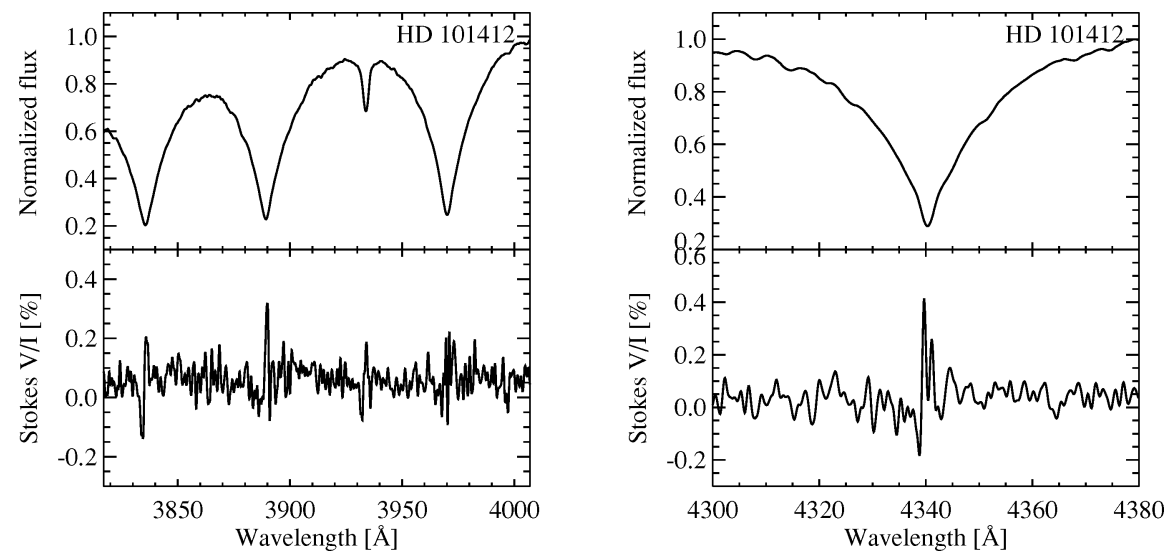

Figure 9. Stokes $I$ and $V$ spectra of the Herbig Ae/Be star HD 101412, with the largest detected magnetic field. Left panel: Zeeman features in $\mathrm{H}_{9}, \mathrm{H}_{8}$, Ca II $\mathrm{H} \& \mathrm{~K}$, and $\mathrm{H} \epsilon$ profiles. Right panel: Stokes $I$ and $V$ spectra in the vicinity of the $\mathrm{H} \gamma$ line.

Küker (2009), who considered a dynamo process of $\alpha^{2}$-type. The non-linear induction equation was solved in three dimensions to allow for non-axisymmetric solutions. The resulting magnetic field was found purely non-axisymmetric and steady (see Fig. 8). Two years later Kitchatinov (2001) carried out simulations of a convection zone dynamo and an internal field captured by a growing radiative core for a pre-main sequence solar mass star. His computations suggest that an infant Sun with its core just starting to grow generates a non-axisymmetric global field.

The quite recent work of Sokoloff et al. (2008) was based on a representation of the stellar magnetic field as a superposition of a finite number of poloidal and toroidal free damping modes. Assuming a "realistic" law of stellar differential rotation the authors found that a dynamo starts to work in fully convective stars when the dynamo number D $>5 \times 10^{3}$.

The presence of magnetic fields in Herbig Ae stars has long been suspected, in particular on account of $\mathrm{H} \alpha$ spectropolarimetric observations pointing out the possibility of the existence of a physical transition region in the H-R diagram from magnetospheric accretion, similar to that of classical T Tau stars (e.g. Vink et al. 2002). In the course of our spectropolarimetric studies of Herbig Ae stars (Hubrig et al. 2004; Hubrig et al. 2006c; Hubrig et al. 2007; Hubrig et al. 2009c) we have for the first time examined the relation of the measured field strengths to various parameters that characterize the star-disk system.

In our sample, the Herbig Ae star HD 101412 exhibited the largest magnetic field strength: $\left\langle B_{\mathrm{z}}\right\rangle=-454 \pm 42 \mathrm{G}$. In Fig. 9 we present distinct Zeeman features detected at the positions of the hydrogen Balmer lines and the Ca II H\&K lines.

In Fig. 10, on the left side, we present the strength of the magnetic field plotted versus $\log L_{\mathrm{X}}$. We find a hint for an increase of the magnetic field strength with the level of the X-ray emission, also supported by the correlation analysis which yields a $>90 \%$ probability that a correlation is present between $\left\langle B_{\mathrm{z}}\right\rangle$ and $\log L_{\mathrm{X}}$. This could suggest a dynamo mechanism responsible for the coronal activity in Herbig Ae stars.

There are also clear indications for a trend towards stronger magnetic fields in younger Herbig Ae stars (Fig. 10, right side). The magnetic fields become very weak at the end of their PMS life. Similarly, strong X-ray sources are only found at the youngest ages, in qualitative agreement with the predictions of a shear dynamo that decays within a few 

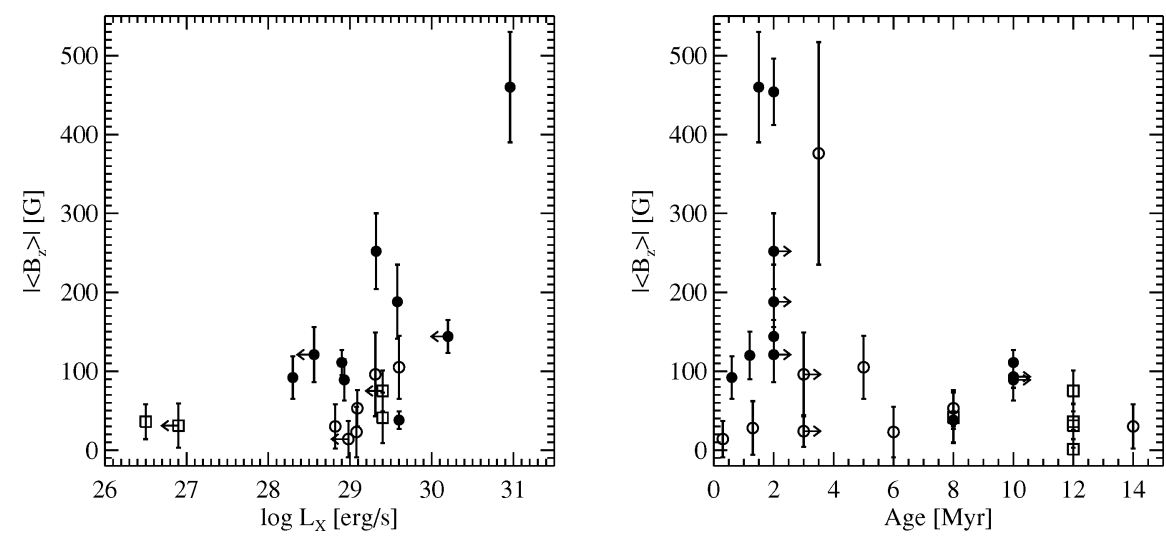

Figure 10. Left panel: The strength of the longitudinal magnetic field plotted over the X-ray luminosity. Right panel: The strength of the longitudinal magnetic field as a function of age. Filled circles denote Herbig Ae stars with a $3 \sigma$ magnetic field detection, while open circles denote Herbig Ae stars with non-detections. Squares denote stars with debris disks, of which none has a $3 \sigma$ magnetic field detection.

Myrs as the rotational energy of the star decreases (Tout \& Pringle 1995). Furthermore, the stars seem to obey the universal power-law relation between magnetic flux and X-ray luminosity established for the Sun and main-sequence active dwarf stars (Pevtsov et al. 2003).

\section{Summary}

Dynamo models have undergone considerable progress in recent years, evolving from the solar dynamo to the larger class of stellar dynamos. To better understand the magnetic field generation in various types of stars it is necessary to obtain in future observations more information on activity, magnetic fields and magnetic field topology in stars of different mass and at different age. Testing the present dynamo models against observational constraints is crucial to identify necessary steps for the further development of dynamo theories.

\section{References}

Arlt, R., Hollerbach, R., \& Rüdiger, G. 2003, A\&A, 401, 1087

Barnes, S. A. 2007, ApJ, 669, 1167

Browning, M. K. 2008, ApJ, 676, 1262

Cantiello, M., Langer, N., Brott, I., et al. 2009, A\& $A$, 499, 279

Chabrier, G. \& Küker, M. 2006, A\&A, 446, 1027

Donati, J.-F., Babel, J., Harries, T. J., et al. 2002, MNRAS, 333, 55

Donati, J.-F., Howarth, I. D., Bouret, J., et al. 2006a, MNRAS, 365, 6

Donati, J.-F., Howarth, I. D., Jardine, M. M., et al. 2006b, MNRAS, 370, 629

Dorch, S. B. F. 2004, A\&A, 423, 1101

Featherstone, N. A., Browning, M. K., Brun, A. S., et al. 2007, AN, 328, 1126

Gilliland, R. L. \& Dupree, A. K. 1996, ApJ (Letters), 463, L29

Hempelmann, A., Schmitt, J. H. M. M., Schultz, M., et al. 1995, A\&A, 294, 515

Hubrig, S., Plachinda, S. I., Hünsch, M., et al. 1994, A\&A, 291, 690

Hubrig, S. \& Mathys, G. 1995, ComAp, 18, 167

Hubrig, S. 1998, CoSka, 27, 249

Hubrig, S., Schöller, M., \& Yudin, R. V. 2004, A\&A (Letters), 428, L1 
Hubrig, S., Briquet, M., Schöller, M., et al. 2006a, MNRAS, 369, 61

Hubrig, S., González, J. F., Savanov, I., et al. 2006b, MNRAS, 371, 1953

Hubrig, S., Schöller, M, Yudin, R. V., et al. 2006c, A\&A, 446, 1089

Hubrig, S., Pogodin, M., Yudin, R. V., et al. 2007, A\&A, 463, 1069

Hubrig, S., Schöller, M., Schnerr, R. S., et al. 2008a, A\&\&A, 490, 793

Hubrig, S., Briquet, M., Morel, T., et al. 2008b, A\& $A$, 488, 287

Hubrig, S., Briquet, M., Morel, T., et al. 2009a, AN, 330, 317

Hubrig, S., Schöller, M., Savanov, I., et al. 2009b, AN, 330, 708

Hubrig, S., Stelzer, B., Schöller, M., et al. 2009c, $A \mathscr{\xi} A, 502,283$

Hünsch, M., Schmitt, J. H. M. M., \& Voges, W. 1998, A\& AS, 127, 251

Kitchatinov, L. L. 2001, ARep, 45, 816

Konstantinova-Antova, R., Auriére, M., Iliev, I. Kh., et al. 2008, A\&AA, 480, 475

Küker, M. \& Rüdiger, G. 1999, A\&\&A, 346, 922

Küker, M. 2009, AIPC, 1094, 77

MacGregor, K. B. \& Cassinelli, J. P. 2003, ApJ, 586, 480

Mamajek, E. E. \& Hillebrand, L. A. 2008, ApJ, 687, 1264

McKee, C. F. \& Ostriker, E. C. 2007, ARAA, 45, 565

Montesinos, B., Thomas, J. H., Ventura, P., et al. 2001, MNRAS, 326, 877

Morin, J., Donati, J.-F., Petit, P., et al. 2008, MNRAS, 390, 567

Noyes, R. W., Weiss, N. O., \& Vaughan, A. H. 1984, ApJ, 287, 769

Pevtsov, A. A., Fisher, G. H., Acton, L. W., et al. 2003, ApJ, 598, 1387

Reiners, A. \& Basri, G. 2009, A\&SA, 496, 787

Robrade, J. \& Schmitt, J. H. M. M. 2009, A\&A, 497, 511

Rüdiger, G. \& Kitchatinov, L. L. 2009, astro-ph/09081841

Savanov, I. S., Hubrig, S., González, J. F., et al. 2009, IAUS, 259, 401

Sivagnanam, P. 2004, MNRAS, 347, 1084

Skumanich, A. 1972, ApJ, 171, 565

Stothers, R. B. \& Chin, C. 1993, ApJ (Letters), 408, L85

Soderblom, D. R., Duncan, D. K., \& Johnson, D. R. H. 1991, ApJ, 375, 722

Sokoloff, D. D., Nefedov, S. N., Ermash, A. A., et al. 2008, AstL, 34, 761

Tout, C. A. \& Pringle, J. E. 1995, MNRAS, 272, 528

Vink, J. S., Drew, J. E., Harries T. J., et al. 2002, MNRAS, 337, 356 